\title{
Slow Pathway Ablation for Atrioventricular Nodal Reentry Using a Right Internal Jugular Vein Approach: A Case Series
}

\author{
YASSER S. SALEM, * MARIN C. BURKE, * SUSAN S. KIM,* FRED MORADY,† \\ and BRADLEY P. KNIGHT* \\ From the *Division of Cardiology, Department of Internal Medicine, University of Chicago, Chicago, Illinois, and \\ tDivision of Cardiology, Department of Internal Medicine, University of Michigan, Ann Arbor, Michigan
}

\begin{abstract}
SALEM, Y.S., ET AL.: Slow Pathway Ablation for Atrioventricular Nodal Reentry Using a Right Internal Jugular Vein Approach: A Case Series. Background: Inferior venous access to the right heart is not possible in some patients due to congenital or acquired obstruction of the inferior vena cava (IVC). Although rightsided electrophysiology procedures have been performed successfully in patients with a previously placed IVC filter by direct placement of catheters through the filter, an alternative approach is necessary in some patients.
\end{abstract}

Methods: This case series describes three patients with an IVC filter who underwent successful ablation of the slow pathway for typical atrioventricular (AV) nodal reentrant tachycardia using a superior vena cava (SVC) approach via the right internal jugular (IJ) vein. Two separate introducer sheaths were placed into the IJ vein using separate punctures. This permitted placement of a standard deflectable ablation catheter and an additional catheter in the right atrium to monitor for ventriculoatrial conduction during the junctional rhythm associated with ablation of the slow AV nodal pathway.

Results: Catheter ablation was successful in each patient. The number of radiofrequency current applications was 7,17 , and 27. There were no procedural complications and no patient had recurrent tachycardia during follow-up.

Conclusions: Catheter ablation of the slow AV nodal pathway can be performed successfully and safely in patients with inferior venous barriers to the right heart using an SVC approach via the right IJ vein. (PACE 2006; 29:59-62)

\section{atrioventricular nodal reentrant tachycardia, catheter ablation}

\section{Introduction}

Radiofrequency (RF) catheter ablation of the slow atrioventricular (AV) node pathway is an effective treatment for AV nodal reentrant tachycardia (AVNRT). ${ }^{1}$ For ablation of right-sided cardiac structures, ablation catheters are usually placed through a femoral vein. However, inferior venous access to the right heart is not possible in some patients due to congenital or acquired vena caval obstruction. ${ }^{2}$ Although right-sided electrophysiology (EP) procedures have been performed successfully in patients with a previously placed inferior vena cava (IVC) filter by direct placement of catheters through the filter, ${ }^{3}$ an alternative approach is necessary in some patients. This case series describes three patients with an IVC filter who underwent successful ablation of the slow pathway for typical AVNRT using a superior vena cava

\footnotetext{
Address for reprints: Bradley P. Knight, M.D., Center for Advance Medicine, University of Chicago Hospitals, MC 9024, 5758 South Maryland Avenue, Chicago, IL 60637. Fax:773-7024666; e-mail: bknight@medicine.bsd.uchicago.edu

Received August 24, 2005; revised October 10, 2005; accepted October 12, 2005.
}

(SVC) approach via the right internal jugular (IJ) vein.

\section{Methods}

Three patients with medically refractory, recurrent paroxysmal supraventricular tachycardia (PSVT) underwent a catheter ablation procedure. Tachycardia had been documented in each patient using a 12-lead ECG and was consistent with typical AVNRT. A catheter ablation procedure was performed in each patient using an SVC approach because each had previously undergone insertion of an IVC filter for recurrent venous thromboembolism.

The ablation procedure was performed in the fasting state after antiarrhythmic drugs had been held for at least five half-lives. A modified Seldinger technique was used to insert two introducer sheaths ( $7 \mathrm{Fr}$ and $6 \mathrm{Fr}$ ) into the right IJ vein using separate needle punctures. A quadrapolar electrode catheter was advanced through one of the sheaths into the right atrium. A deflectable ablation catheter with a 4-mm tip electrode (Boston Scientific Inc., Sunnyvale, CA) was advanced into the right heart through the remaining sheath and positioned near the His-bundle region (Fig. 1).

(C)2006, The Authors. Journal compilation (C)2006, Blackwell Publishing, Inc. 


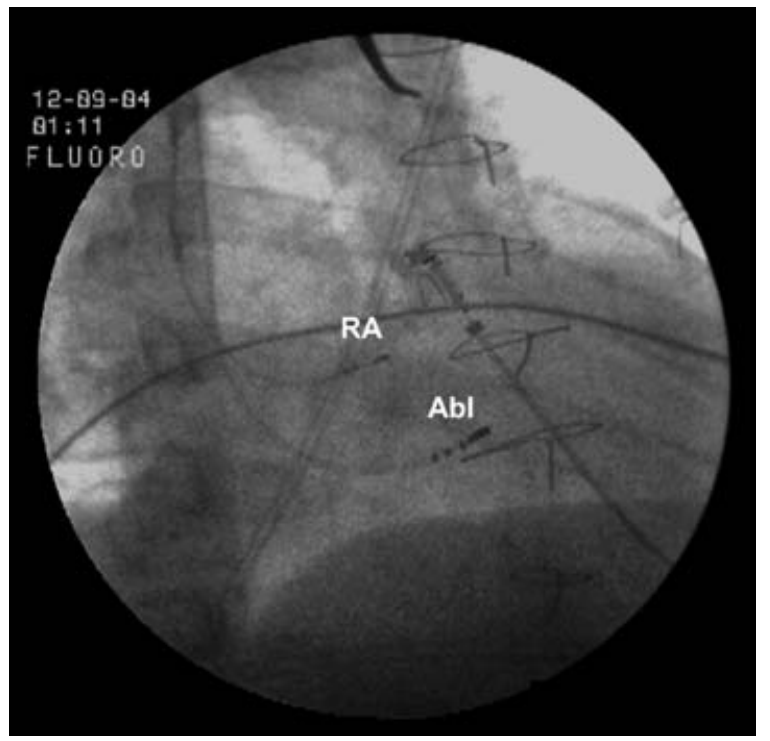

Figure 1. Fluoroscopic view of two quadripolar electrode catheters placed via the right internal jugular vein. The ablation catheter (Abl) is shown near the His-bundle region and the quadripolar catheter is in the right atrium (RA). Sternotomy wires can be seen from prior cardiac transplantation. The view is right anterior oblique.

Intravenous unfractionated heparin, 3,000 unit bolus followed by a 1,000 unit bolus every hour, was administered after vascular access was obtained. Intracardiac signals were processed and displayed. An advanced mapping system was not used.

The baseline rhythm was sinus without ventricular preexcitation in each patient. Supraventricular tachycardia was induced with atrial pacing. The septal ventriculoatrial (VA) interval during tachycardia was less than $70 \mathrm{~ms}$ for each patient, excluding orthodromic AV reentry as the mechanism. Atrial tachycardia was excluded based on observations that induction of the tachycardia was dependent on a long AH interval and that spontaneous termination was associated with AV block. Therefore, the tachycardia mechanism was determined to be typical AV nodal reentry.

The tip of the ablation catheter was repositioned to the posterior right atrial septum near the upper aspect of the coronary sinus ostium using fluoroscopy (Fig. 2). The ablation electrode was stabilized at a target site that was associated with a fractionated, multicomponent atrial electrogram and an AV ratio between 1:4 and 1:8. RF current was delivered using a RF generator with an automatic temperature feedback control set to a maximum power of $50 \mathrm{~W}$ and a maximum temperature of $52^{\circ} \mathrm{C}-60^{\circ} \mathrm{C}$ for a maximum duration of 60 seconds. Delivery of RF energy at each suc-

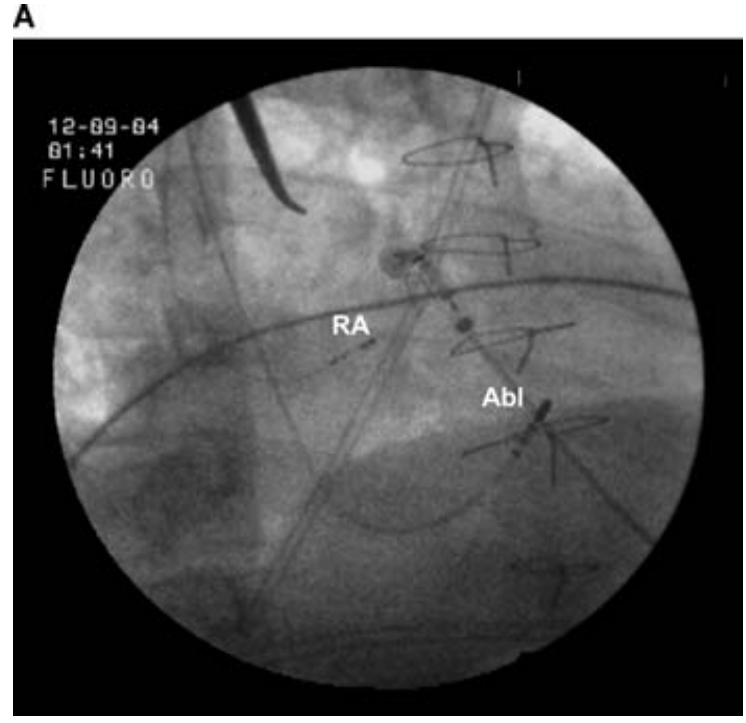

B

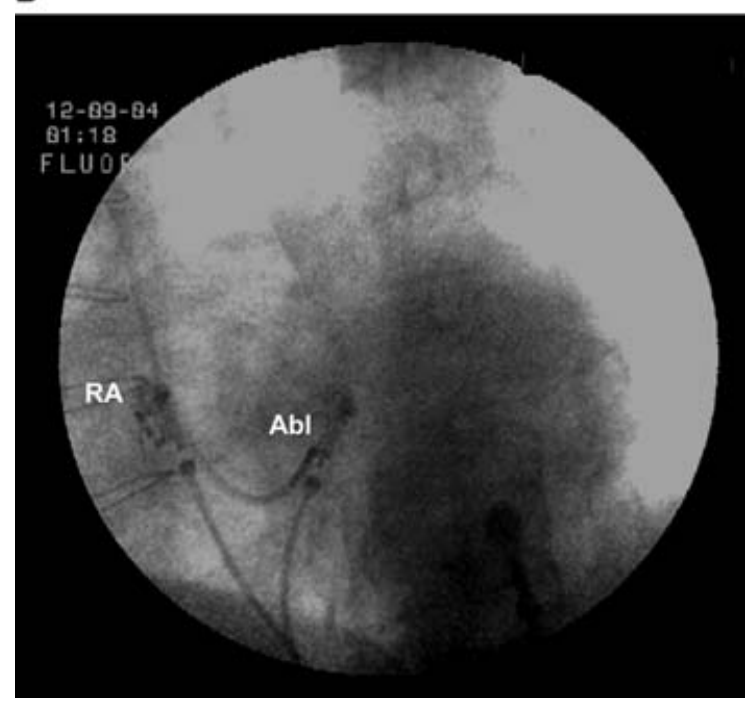

Figure 2. Fluoroscopic views of the catheter locations during successful radiofrequency ablation of the slow $A V$ nodal pathway in the same patient shown in Figure 1. The ablation catheter (Abl) has been repositioned from its initial location shown in Figure 1 to the posterior right atrial septum near the superior aspect of the coronary sinus ostium. The right atrial (RA) catheter is used to monitor ventriculoatrial conduction during the junctional rhythm associated with successful slow pathway ablation. The right anterior oblique view is shown in panel $A$ and the left anterior oblique view is shown in panel $B$.

cessful site was associated with an accelerated junctional rhythm (Fig. 3). Energy delivery was terminated if VA conduction block was observed during the junctional rhythm. Programmed stimulation was repeated after ablation with and without 

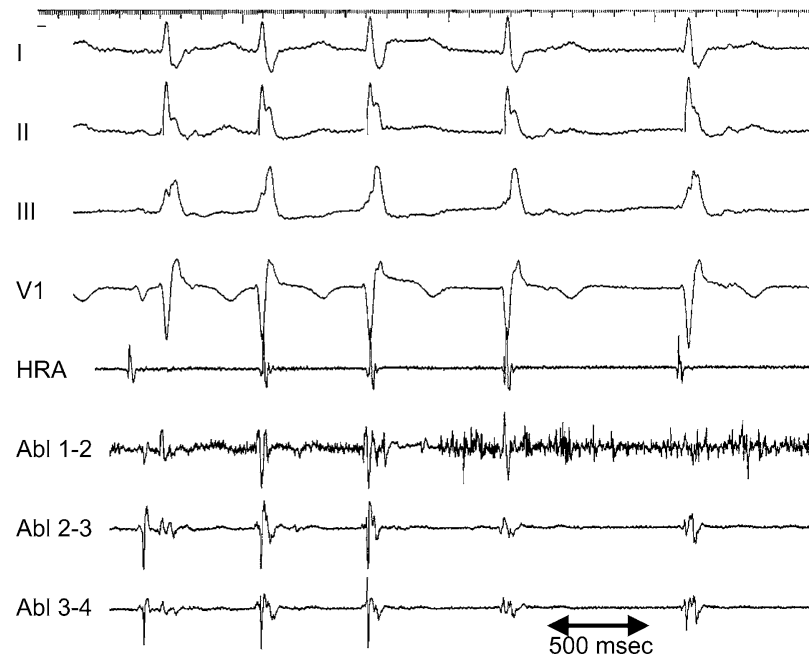

Figure 3. Recording of an accelerated junctional rhythm during application of radiofrequency current at the successful slow pathway ablation site during an ablation via a right internal jugular vein. Shown are surface leads I, II, III, V1, and the intracardiac recordings from the high right atrium (HRA), and the ablation catheter (Abl) distal, middle, and proximal electrode pairs.

isoproterenol infusion. The endpoint for ablation was either complete elimination or modification of slow pathway conduction and noninducibility. Sheaths were removed immediately after the procedure. There were no complications and no recurrent tachycardia during follow-up.

\section{Case 1}

A 52-year-old white woman presented with a 5 -year history of medically refractory SVT. She had a history of deep venous thrombosis and had undergone placement of an IVC filter 3 years prior to presentation. During her EP procedure, sustained typical AVNRT was induced at a cycle length of $300 \mathrm{~ms}$. Successful ablation of the slow pathway was performed with seven RF applications. Total RF delivery time was 6 minutes and 42 seconds. The total procedure time was 50 minutes and total fluoroscopy time was 18 minutes.

\section{Case 2}

A 55-year-old woman was referred for evaluation after 10 years of drug-refractory PSVT that had required multiple visits to the emergency department. Her tachycardia terminated either spontaneously or after administration of AV nodal blocking agents. She had a hypercoagulable state and had undergone placement of an IVC filter 5 years prior to her ablation procedure for lower extremity deep venous thrombosis. She also had bilateral subclavian vein thrombosis. Her tachycardia was refractory to several anti-arrhythmic drugs including amiodarone. During the EP procedure, typical AVNRT was inducible at a cycle length of $320 \mathrm{~ms}$. The patient underwent successful slow pathway ablation after a total of 27 applications of RF energy. The total procedure time was 90 minutes and total fluoroscopy time was 40 minutes.

\section{Case 3}

A 49-year-old man presented with a short RP tachycardia at a rate of 180 beats/min which terminated with intravenous adenosine. He had undergone orthotopic heart transplantation 10 years prior to his ablation procedure with an atrioatrial anastomosis. His postoperative course was complicated by deep venous thrombosis and pulmonary embolism and he was treated with anticoagulation and Greenfield IVC filter placement. His tachycardia was recurrent despite treatment with diltiazem and digoxin.

During his EP procedure, dual AV nodal physiology was present at baseline and sustained typical AVNRT was induced at a cycle length of $245 \mathrm{~ms}$. Successful ablation of the posterior slow pathway was performed with $17 \mathrm{RF}$ applications. Total RF delivery time was 6 minutes and 42 seconds. The total procedure time was 105 minutes and total fluoroscopy time was 15 minutes.

\section{Discussion}

This case series demonstrates that the SVC approach via the right IJ vein can be a successful alternative approach for catheter ablation of the slow AV nodal pathway in patients with AVNRT. This technique should be considered in patients with obstruction of the IVC.

The presence of an IVC filter does not appear to be an absolute contraindication to a femoral vein approach for catheter ablation. There are several reports of successful passage of luminal catheters across an IVC filter for right heart catheterization, ${ }^{4-6}$ and there was a recent report of successful placement of electrode catheters across an IVC filter for catheter ablation of typical atrial flutter. ${ }^{3}$ When preparing to pass catheters across an IVC filter, it is reasonable to perform venography of the IVC beforehand to assure patency and to examine the filter fluoroscopically to confirm that the fenestrations are large enough to accommodate the catheters. Despite these successes, complications have also been reported as a consequence of crossing an IVC filter, such as device migration, dislodgment, and fracture, and entrapment of a guidewire or catheter within the filter. ${ }^{7}$ The jugular approach avoids the potential complications associated with crossing an IVC filter.

In some patients, the IVC or bilateral femoral veins are completely occluded and an inferior 
venous approach to the right heart is not feasible. Alternative methods of right heart catheterization include the use of the jugular, subclavian, basilic, or azygous veins. Avella et al. described the successful ablation of a slow AV nodal pathway in two patients via a superior approach. ${ }^{8}$ Electrode catheters were placed via the left subclavian and right basilica vein in each patient. In one patient, a third electrode catheter was placed from the femoral vein into the right atrium via a large azygous vein that emptied into the SVC. There are two additional case reports of successful ablation of AVNRT using a superior approach. In each case, two catheters were placed via a subclavian vein. ${ }^{9,10}$ In one of the two cases, however, catheter tip stability was inadequate at the slow pathway region and a fast pathway ablation was required for successful elimination of the tachycardia. ${ }^{10}$ A case was also recently reported that describes successful ablation of the cavotricuspid isthmus for atrial flutter in a patient with anomalous IVC and azygous continuation using a subclavian vein approach. ${ }^{11}$ The present cases are the first reports of the use of the IJ vein for slow pathway ablation.

There are advantages and disadvantages to the use of a superior approach compared to the conventional femoral approach for catheter ablation of the slow AV nodal pathway. Successful slow pathway ablation requires stable positioning of the ablation catheter near the triangle of Koch. Using a typical femoral vein approach, the Eustachian ridge can make it difficult to position the ablation electrode toward the posterior right atrial septum. An advantage of the superior approach is that it permits the shaft of the catheter to be superior to the Eustachian ridge when the tip of the catheter is at the posterior right atrial septum. Disadvantages of the superior approach include potential for greater radiation exposure to the ablationist due to a closer proximity of the operator to the image intensifier, and the challenge of manipulating a catheter and viewing intracardiac electrograms and fluoroscopy images from an unconventional angle. The relatively high number of RF applications required in the cases presented (between 7 and 27 applications) also argues against the routine use of the superior approach for slow pathway ablation.

Monitoring VA conduction during the junctional rhythm associated with slow pathway ablation is critical to avoid inadvertent AV block. Therefore, to safely perform a slow pathway ablation, an electrode catheter in the right atrium is necessary in addition to the ablation catheter. To avoid the need to access multiple veins, multiple introducer sheaths are commonly inserted into a single femoral vein. However, it is uncommon to place more than one introducer sheath in an IJ vein. These cases demonstrate that two central venous sheaths, large enough to accommodate an ablation catheter and an additional pacing electrode catheter, can easily be placed percutaneously via the right IJ vein. Instead of placing a quadripolar catheter in the high right atrium, a decapolar catheter could have been placed in the coronary sinus or a single multipolar catheter could have been positioned across the tricuspid valve with electrodes spanning the annulus. These alternative catheter positions would have allowed for simultaneous recordings from the atrium and ventricle with one catheter and might have permitted pacing maneuvers to be performed from both the atrium and the ventricle.

\section{Conclusions}

Catheter ablation of the slow AV nodal pathway can be performed successfully and safely in patients with inferior venous barriers to the right heart using the SVC approach via the right IJ vein.

\section{References}

1. Jackman WM, Beckman KJ, McClelland JH, et al. Treatment of supraventricular tachycardia due to atrioventricular nodal reentry, by radiofrequency catheter ablation of slow-pathway conduction. N Engl J Med 1992; 327:313-318.

2. Debing E, Tielemans Y, Jolie E, Van den Brande P. Congenital absence of inferior vena cava. Eur J Vasc Surg 1993; 7:201-203.

3. Sinha SK, Harnick D, Gomes JA, Mehta D. Electrophysiologic interventions in patients with inferior vena cava filters. Safety and efficacy of the transfemoral approach. Heart Rhythm 2005; 2:15-18.

4. Hansen ME, Geller SC, Yecel EK, Egglin TK, Waltman AC. Transfemoral venous catheterization through inferior vena cava filters: Results in seven cases. Am J Roentgenol 1992; 159:434-435.

5. Kussmaul WG, Secaira R, McCormick DJ, Cohen M. Right heart catheterization in the presence of an inferior vena cava filter. Catheter Cardiovasc Interv 2001; 52:476-478.

6. Rhodes JF, Miller SG, Lane GK, Latson LA. Transcatheter interventions across an inferior vena cava filter. Catheter Cardiovasc Interv 2003; 59:333-337.

7. Streib EW, Wagner JW. Complications of vascular access procedures in patients with vena cava filters. J Trauma 2000; 49:557558.

8. Avella A, De Ponti R, Tritto M, Spadacini G, Salerno-Uriarte JA. Radiofrequency catheter ablation of atrioventricular nodal reentry tachycardia: Selective approach to slow pathway via superior vena cava. Ital Heart J 2001; 142-146.

9. Chandan K, Ponde C, Lokhandwala Y, Modhe J. Slow pathway ablation without femoral access. Indian Heart J 2002; 54:8082.

10. Machado C, Liddon V, Roy D. Radiofrequency catheter ablation of fast pathway via unconventional right subclavian venous access for atrioventricular nodal reentrant tachycardia. J Interv Cardiol 2003; 16:377-380.

11. Malavasi VL, Casali E, Rossi L, Modena MG. Radiofrequency catheter ablation of common atrial flutter in a patient with anomalous inferior vena cava and azygous continuation. Pacing Clin Electrophysiol 2005; 28:733-735. 